\title{
Infant feeding practices and breastfeeding duration in Japan: A review
}

Madoka Inoue ${ }^{1}$, Colin W Binns ${ }^{1 *}$, Keiko Otsuka ${ }^{2}$, Masamine Jimba ${ }^{2}$ and Manami Matsubara ${ }^{3}$

\begin{abstract}
The Japanese health system places great emphasis on healthy development. However, the prevalence of Exclusive Breastfeeding at one month postpartum between 1980 and 2005 has remained unchanged, fluctuating between $42 \%$ and $49 \%$. At the same time, the Any Breastfeeding prevalence has gradually increased from about $80 \%$ to $95 \%$. In 2010, the latest national breastfeeding report showed that 'exclusive' and 'any' breastfeeding rates have improved. However, as the World Health Organization (WHO) definition of breastfeeding practices was not used in this study or in other national surveys, it is difficult to interpret these latest results. While the Japanese government has launched several promotion projects, there have been few studies and reviews of risk factors that influence breastfeeding duration. The objectives of this review were to summarise the factors that have influenced the duration of breastfeeding in Japan to provide information relevant to breastfeeding promotion programs. A search of electronic databases in Japanese and English was undertaken up to 2011. The inclusion criteria for this review were studies that focused on infant feeding practices and targeted Japanese mothers, fathers, or health professionals, but excluded mothers' friends and peer groups. In total, 12 articles were selected for the final analysis. Smoking status, low birth weight of infants and maternal perceptions of insufficient breast milk supply were negative influences on breastfeeding duration, while support from husbands/partners is associated with continued breastfeeding. Some factors that have been found to be associated with breastfeeding in other countries, including maternal age, family income, maternal educational levels, and living with grandparents of infants have not been confirmed in Japan. While the national breastfeeding rates were higher than other countries of similar health status, inconsistent knowledge of breastfeeding benefits and inappropriate hospital practices remain in Japan may be associated with increased the use of infant formula and reduced breastfeeding duration. Most of the studies reviewed were cross-sectional in design, with only a limited number of cohort studies. Also many published studies used small sample sizes. Cohort studies of infant feeding practices with larger sample sizes are required to monitor trends in rates and risk factors for breastfeeding outcomes.
\end{abstract}

\section{Background}

The importance of breastfeeding has been documented in numerous scientific studies, including recent major reviews [1-3]. Breastfeeding, particularly Exclusive Breastfeeding for the initial six months of life, provides better health for both infants and mothers by preventing diseases and promoting health in the short and long term [4]. There has been an emphasis on the promotion of breastfeeding in many countries and in 2001 the Japanese government undertook a health promotion project, 'Healthy and Happy Family 21 (Sukoyaka Oyako 21)'

\footnotetext{
* Correspondence: C.Binns@curtin.edu.au

${ }^{1}$ School of Public Health, Curtin Health Innovation Research Institute, Curtin University, GPO Box U1987, Perth, Western Australia 6845, Australia Full list of author information is available at the end of the article
}

that included a goal to improve the rate of Exclusive Breastfeeding at one month postpartum by 2014. The 2010 interim report on this project stated that although more than $95 \%$ of women intended, before delivery, to breastfeed their infants, the rate of Exclusive Breastfeeding at one month postpartum had not changed since $2005[5,6]$. No specific target was set at the beginning of this project, making it hard for health professionals to evaluate if the goal was achieved. Subsequently in 2010, a target was set of $60 \%$ Exclusive Breastfeeding at one month postpartum [5].

In Japan, Exclusive Breastfeeding was the only practical feeding method to feed infants until the end of the first half of the 20th century [7]. For women, visiting temples or shrines to pray for their ability to have

\section{Biomed Central}


sufficient production of breastmilk was common practice and many special foods were consumed to help with lactation [8]. A child usually continued breastfeeding until two to three years of age, and breastfeeding up to six years of age was common [7]. Infant formula was first introduced to Japan in 1917, but it was not until the 1950s that its use increased rapidly [9]. Formula companies used the advertising slogan 'To become a bright child' in their marketing, and this message suggested that infant formula feeding gave advantages in infant development and weight gain, which increased its popularity among mothers [10]. The Exclusive Breastfeeding rate declined to its lowest point of $31 \%$ at one month in 1970 [Figure 1]. As a result, in 1975, three goals for restoring breastfeeding rates were set by the Ministry of Welfare based on the 1974 recommendations of the WHO. These were:

1) To exclusively breastfeed infants to 1.5 months after birth

2) To exclusively breastfeed infants to three months if possible, and

3) Not to change to formula, even after four months of age, without good reasons [11]

The Any Breastfeeding rate has gradually increased since then. The National Nutritional Survey of Preschool Children, which has been carried out every decade since 1985, showed that Any Breastfeeding has increased from $80.7 \%$ in 1980 to $94.9 \%$ in 2005 at one month and from $59.5 \%$ to $79.0 \%$ at three months, while the duration of Exclusive Breastfeeding has remained relatively constant, but with a slight decline in recent years [Figures 1 and 2] [6]. The rate of Exclusive Breastfeeding at one month declined from $49.5 \%$ in 1985 to
$46.2 \%$ in 1995 and to $42.2 \%$ in 2005 [Figure 1] [6]. The rate at three months has also remained relatively constant at $39.5 \%$ in $1985,38.1 \%$ in 1995 and $38.0 \%$ in 2005 [Figure 2] [6]. In 2010, another National Survey on Children's Growth reported that the rate of Exclusive Breastfeeding was $51.6 \%$ at one month [Figure 1] and 56.8\% at three months [Figure 2] [13]. In this survey, Any Breastfeeding also increased to $95.4 \%$ at one month and $86.8 \%$ at three months. Compared with previous reports of breastfeeding rates in Japan, the latest rates of Exclusive Breastfeeding have improved. However, the WHO definitions of breastfeeding practices were not applied in this study and therefore it is difficult to compare 2010 results with international studies. While the Japanese government set three goals for mothers in the first breastfeeding promotion campaign in 1975 (see above), the rate of Exclusive Breastfeeding has remained unchanged for the last 30 years.

Japan was the first developed country to have a BabyFriendly Hospital Initiative (BFHI) hospital accredited by the WHO and United Nations Children's Fund (UNICEF) in 1991 [14]. However, Japan was also one of the three countries that abstained from voting for 'International Code of Marketing of Breastmilk Substitutes' in 1981 [9]. These contradictive actions suggested to Japanese health professionals that perhaps breastfeeding promotion was not a high priority and not an issue that should be applied to the whole population. Studies from other countries have found that factors that influence breastfeeding outcomes include maternal age, maternal infant feeding attitudes, breastfeeding difficulties, maternal employment status, and maternal smoking status [15-17]. However, there have been few studies published on the factors associated with breastfeeding duration in Japan. The aim of this review is to summarise factors

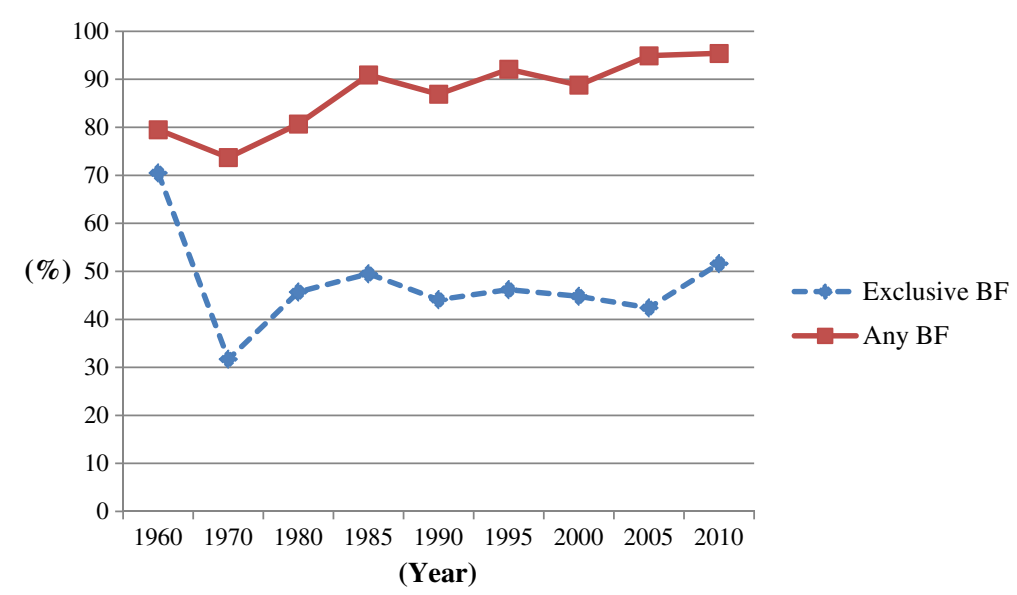

Figure 1 The breastfeeding rate at one month after birth in Japan from 1960 to 2010. Footnote: Source: from 1960 to 2005: [12], 2010:[13]. ${ }^{*} \mathrm{BF}=$ breastfeeding. 'Exclusive Breastfeeding' should be considered as 'full or Predominant Breastfeeding' due to the differing definitions of breastfeeding status used in Japan. 


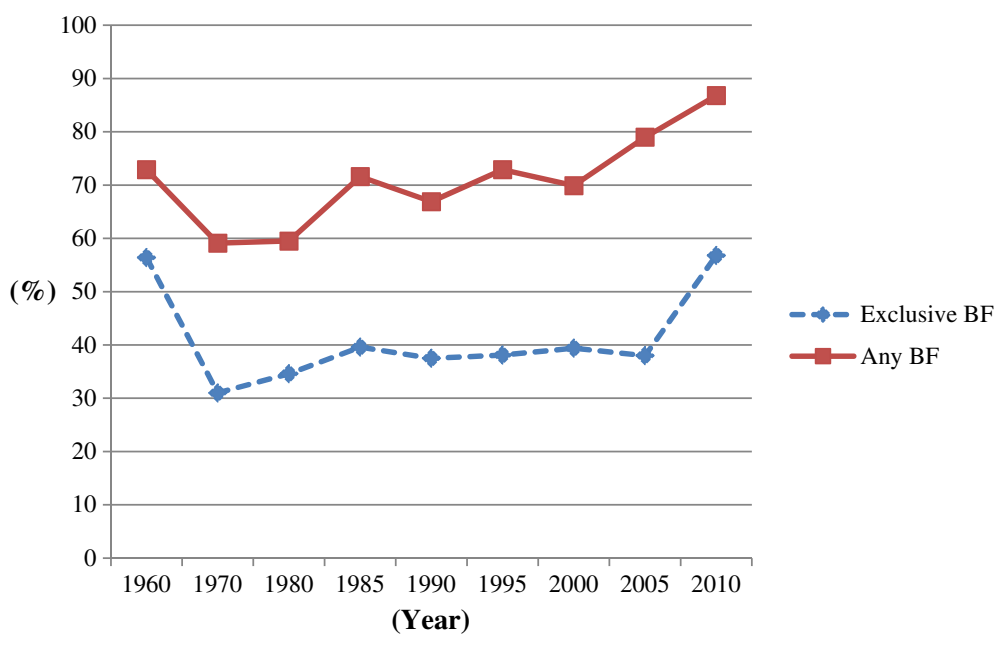

Figure 2 The breastfeeding rate at three months after birth in Japan from 1960 to 2010. Footnote: Source: from 1960 to 2005: [12], 2010: [13]. *BF = breastfeeding. 'Exclusive Breastfeeding' should be considered as 'full or Predominant Breastfeeding' due to the differing definitions of breastfeeding status used in Japan.

that influence the duration of breastfeeding in Japan and the existing knowledge of breastfeeding that will assist the development of further promotion programs.

The standard WHO definitions of breastfeeding used in this review are [18]:

- Exclusive Breastfeeding: Infants receive breastmilk. It precludes the use of any other liquids or solids since delivery, other than specific medications.

- Predominant (full) Breastfeeding: Infants receive breastmilk as the main nourishment and are also allowed to have water, water-based drinks, fruits juice, and ORS. However, it precludes the use of formula and solids.

- Any (complementary) Breastfeeding: Infants receive some breastmilk and may also receive infant formula with or without solids.

\section{Methods}

A literature search was undertaken using the electronic databases, PubMed, Proquest, Web of Science, ICHUSHI, J-STAGE, and CiNii using the key-words of 'breastfeeding,' 'breast-feeding,' 'factor(s),' 'determinant(s)', 'breastfeeding duration' 'fathers (spouse/partners)' 'Japan', 'Japanese', and 'infant feeding'. These key-words were used in combination with the Boolean operators 'AND' or 'OR' to search literature and duplicate records were then removed. Irrelevant titles and non-Japanese samples were also removed. After abstracts were reviewed, relevant full-text papers were obtained but irrelevant contents including qualitative studies were again removed. The references in the relevant full-text papers were checked as other sources and included if necessary. The PRISMA 2009 flow diagram [19] describes the process used for searching the relevant literature [Figure 3]. The inclusion criteria for this review were infant feeding practices in Japan and the studies that focused on Japanese mothers, fathers or health professionals. All English and Japanese papers were selected for this review and there were no limitations on the year of publication, to the end of 2011. The exclusion criteria were any papers targeting Japanese mothers' friends.

\section{Results}

In total, 12 articles were reviewed. The factors are categorized into maternal, infant, and socio-environmental attributes. The available data is summarized in Table 1.

\section{Maternal attributes}

Demographic factors that have been studied as risk factors for breastfeeding initiation and duration in Japan have included maternal age, socioeconomic status, maternal education, employment status, delivery method, parity, and smoking habits of mothers and other family members.

While higher maternal age has been associated with a longer duration of breastfeeding in most developed countries [32,33], there is no clear association in Japan. A population based study of 15,262 infants confirmed that the mean age of mothers who chose formula feeding was significantly younger than those who chose 'full' and 'Any Breastfeeding' ( $\mathrm{p}<0.001)$ [21]. This study was cross-sectional and analysed a database of infants aged three to six months attending for medical examinations, which has a very high response rate in Japan. In contrast, a study using data from the National Survey of 46,569 infants showed that mothers in their 30 s and 40 s were less likely to continue Exclusive Breastfeeding at six 


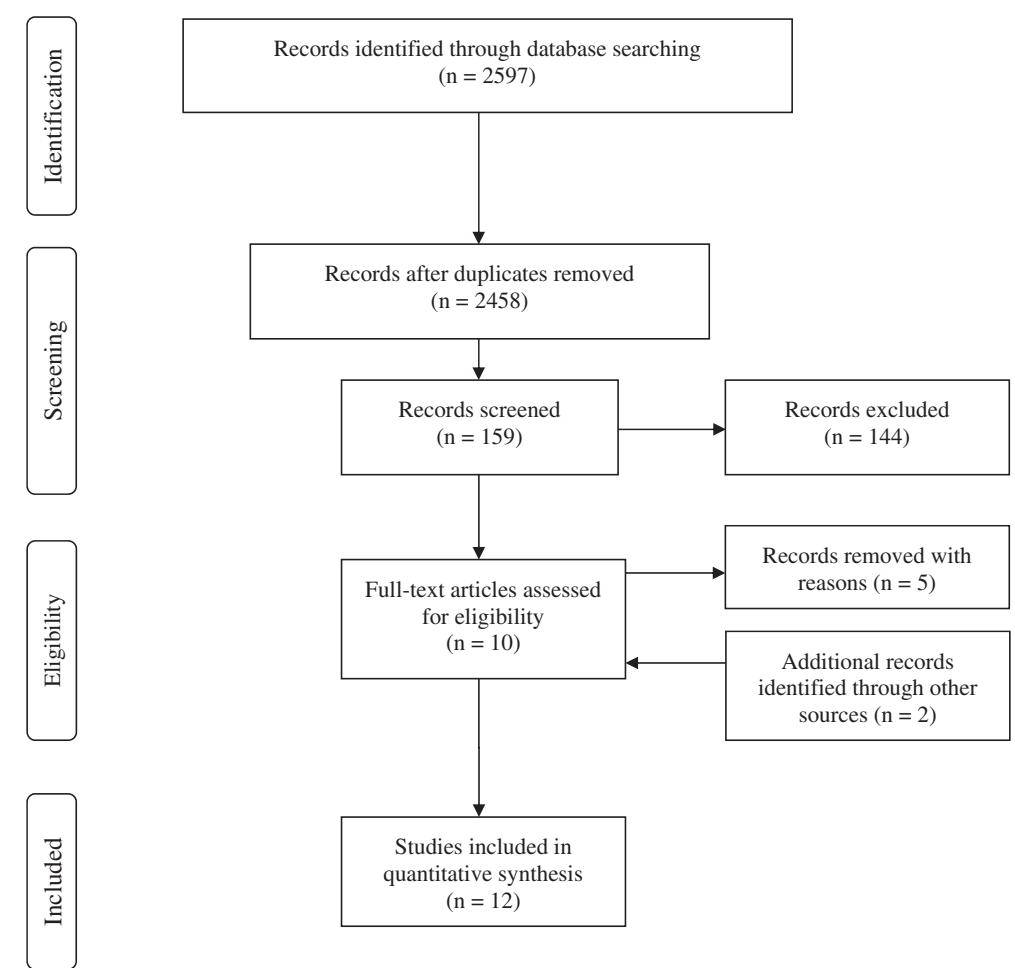

Figure 3 Selection process of the studies reviewed in this paper.

months postpartum than mothers in their 20s $(\mathrm{aOR}=$ $0.89,95 \% \mathrm{CI}=0.84-0.94$ for their $30 \mathrm{~s} ; \mathrm{aOR}=0.56,95 \%$ $\mathrm{CI}=0.48-0.65$ for their 40s, respectively) [20]. The National Survey used a cross-sectional study design and found different results to the study by Yokoyama et al. [21]. Moreover, Sasaki et al. found no correlation between maternal age and Exclusive Breastfeeding at four months postpartum in a longitudinal study $(\mathrm{p}=0.93$, $\mathrm{n}=908$ ) [23]. Otsuka et al. [22] also conducted a longitudinal study $(n=262)$ and the results were different in that older mothers (more than 38 years old) tended to use infant formula at four weeks postpartum ( $<<0.05)$.

Few studies have investigated associations between the duration of breastfeeding and the mothers' socioeconomic and educational status in Japan, which may be related to privacy issues encountered in undertaking this research [34]. A study by Kaneko et al. found that mothers with a lower annual income (less than 3.9 million yen) were less likely to be exclusively breastfeeding at six months $(\mathrm{aOR}=0.93,95 \% \mathrm{CI}=0.88-0.99)$ [20]. A small study of 262 mothers found that educational level was not related to the use of infant formula at one month postpartum $(\mathrm{p}=0.47)$ [22], but large studies would be needed to confirm this result.

Returning to work is a common reason given by mothers to cease breastfeeding [35]. If the mother is the main income earner, this factor is more important [36]. Kaneko et al. showed that Japanese mothers who had full-time jobs and who took childcare leave for more than six months were more likely to continue Exclusive Breastfeeding to six months than unemployed mothers $(\mathrm{aOR}=1.14,95 \% \mathrm{CI}=1.05-1.23)$ [20]. In this study, the mothers who had childcare leave for less than six months or who had not received any childcare leave had less favourable breastfeeding outcomes [20].

Mothers who smoke are less likely to initiate and to continue breastfeeding than those who are non-smokers [37]. Haku and Onishi stated that smoking mothers tended to rely more on formula feeding [38]. Similarly, in the study by Kaneko et al., fewer mothers who smoked at home were still breastfeeding at six months, compared to non-smoking parents $(\mathrm{aOR}=0.44,95 \%$ $\mathrm{CI}=0.34-0.57$ ) [20]. The risk of ceasing breastfeeding by mothers was decreased when only fathers were smokers compared to non-smoking parents $(\mathrm{aOR}=0.92,95 \%$ $\mathrm{CI}=0.88-0.97)[20]$. Kaneita et al. found that the mothers who did not breastfeed their infants were more likely to smoke inside their house, compared with those who were breastfeeding their infants [39]. While this study had a large sample size $(n=44,562)$ and used clear definitions of smoking, breastfeeding duration was not included as a variable and the definition of breastfeeding was unclear.

The majority of women in Japan choose vaginal delivery in contrast to some other Asian countries, including China and Korea, where the number of caesarean 
Table 1 Japanese studies dealing with factors that influence the duration of breastfeeding

\begin{tabular}{|c|c|c|c|c|c|c|c|c|}
\hline Factors & Authors (Year) & Study design & $\begin{array}{l}\text { Sample } \\
\text { size }(n)\end{array}$ & $\begin{array}{l}\text { Definition of } \\
\text { breastfeeding }\end{array}$ & $\begin{array}{l}\text { Exclusive } \\
\text { Breastfeeding } \\
\text { rate }(\%)\end{array}$ & $\begin{array}{l}\text { Crude OR } \\
\text { (95\% Cl) } \\
\text { (subgroup) } \\
\end{array}$ & $\begin{array}{l}\text { Adjusted OR } \\
\text { (95\% CI) } \\
\text { (subgroup) } \\
\end{array}$ & Study details \\
\hline \multirow[t]{12}{*}{ Maternal age } & \multirow{3}{*}{$\begin{array}{l}\text { Kaneko et al. } \\
\text { (2006) [20] }\end{array}$} & \multirow[t]{3}{*}{ Cross-sectional } & \multirow[t]{3}{*}{45,569} & 1. Breastfed & \multirow[t]{3}{*}{22.4 at $6 \mathrm{~m}$} & \multirow{3}{*}{$\begin{array}{l}1.2(1.14,1.26) \\
(30-39 \text { years old })\end{array}$} & \multirow{3}{*}{$\begin{array}{l}0.89(0.84,0.94) \\
(30-39 \text { years old })\end{array}$} & \multirow{9}{*}{$\begin{array}{l}\text { Mothers who are } \\
\text { between } 20-29 \text { years } \\
\text { old = } 1 \text { (reference) }\end{array}$} \\
\hline & & & & 2. Only colostrum & & & & \\
\hline & & & & 3. Not breastfeed & & & & \\
\hline & \multirow{3}{*}{$\begin{array}{l}\text { Yokoyama et al. } \\
\text { (2006) [21] }\end{array}$} & \multirow[t]{3}{*}{ Cross-sectional } & \multirow[t]{3}{*}{15262} & 1. Breastfeeding only & \multirow[t]{3}{*}{ N/A } & \multirow[t]{3}{*}{ N/A } & \multirow[t]{3}{*}{ N/A } & \\
\hline & & & & $\begin{array}{l}\text { 2. Mixed-feeding (mainly } \\
\text { breast with some bottle or } \\
\text { mainly bottle with some } \\
\text { breast) }\end{array}$ & & & & \\
\hline & & & & $\begin{array}{l}\text { 3. Bottle feeding with } \\
\text { formula milk only }\end{array}$ & & & & \\
\hline & \multirow[t]{3}{*}{$\begin{array}{l}\text { Otsuka et al. } \\
\text { (2008) [22] }\end{array}$} & \multirow[t]{3}{*}{ Cross-sectional } & \multirow[t]{3}{*}{262} & $\begin{array}{l}\text { 1. Exclusive Breastfeeding (i.e. } \\
\text { breastmilk only) }\end{array}$ & \multirow[t]{3}{*}{40 at $4 w k s$} & \multirow[t]{3}{*}{$5.37(1.18,24.40)$} & \multirow[t]{3}{*}{ N/A } & \\
\hline & & & & $\begin{array}{l}\text { 2. Partial breastfeeding (i.e. } \\
\text { breastmilk and formula) }\end{array}$ & & & & \\
\hline & & & & $\begin{array}{l}\text { 3. Bottle-feeding (i.e. no } \\
\text { breastmilk at all) }\end{array}$ & & & & \\
\hline & \multirow{3}{*}{$\begin{array}{l}\text { Sasaki et al } \\
\text { (2009) [23] }\end{array}$} & \multirow[t]{3}{*}{ Longitudinal } & \multirow[t]{3}{*}{908} & 1. Only breastfeeding & \multirow[t]{3}{*}{27.6 at $3-4 m$} & \multirow[t]{3}{*}{ N/A } & \multirow[t]{3}{*}{ N/A } & \multirow{3}{*}{$\begin{array}{l}\text { Not statistically } \\
\text { significant }(p=0.93)\end{array}$} \\
\hline & & & & $\begin{array}{l}\text { 2. Breastmilk and } \\
\text { infant formula }\end{array}$ & & & & \\
\hline & & & & 3. Only infant formula & & & & \\
\hline \multirow[t]{3}{*}{ Marital status } & \multirow[t]{3}{*}{$\begin{array}{l}\text { Otsuka et al. } \\
\text { (2008) [22] }\end{array}$} & \multirow[t]{3}{*}{ Cross-sectional } & \multirow[t]{3}{*}{262} & $\begin{array}{l}\text { 1. Exclusive Breastfeeding (i.e. } \\
\text { breastmilk only) }\end{array}$ & \multirow[t]{3}{*}{40 at $4 w k s$} & \multirow[t]{3}{*}{ N/A } & \multirow[t]{3}{*}{ N/A } & $\begin{array}{l}\text { Not statistically } \\
\text { significant }(p=0.88)\end{array}$ \\
\hline & & & & $\begin{array}{l}\text { 2. Partial breastfeeding (i.e. } \\
\text { breastmilk and formula) }\end{array}$ & & & & \\
\hline & & & & $\begin{array}{l}\text { 3. Bottle-feeding (i.e. no } \\
\text { breastmilk at all) }\end{array}$ & & & & \\
\hline Socioeconomic & Kaneko et al. & Cross-sectional & 45,569 & 1. Breastfed & 19 at $6 \mathrm{~m}$ & $0.85(0.80,0.90)$ & $0.93(0.88,0.99)$ & 4.0-7.9 million yen \\
\hline & (2006) [20] & & & 2. Only colostrum & $\begin{array}{l}<3.9 \text { million yen) } \\
22 \text { at } 6 \mathrm{~m} \text { (> } 8.0 \text { million }\end{array}$ & $\begin{array}{l}<3.9 \text { million yen) } \\
1.03(0.97,1.08)\end{array}$ & $\begin{array}{l}\text { < }<3.9 \text { million yen) } \\
1.03(0.97,1.09)\end{array}$ & $\begin{array}{l}\text { Of annual income = } \\
\text { (reference) }\end{array}$ \\
\hline & & & & 3. Not breastfeed & yen) & (> 8.0 million yen) & (> 8.0 million yen) & \\
\hline & $\begin{array}{l}\text { Otsuka et al. } \\
\text { (2008) [22] }\end{array}$ & Cross-sectional & 262 & $\begin{array}{l}\text { 1. Exclusive Breastfeeding (i.e. } \\
\text { breastmilk only) }\end{array}$ & 40 at $4 w k s$ & $\begin{array}{l}1.0(0.26,3.55) \\
(>2,000,000 \text { yen })\end{array}$ & N/A & $\begin{array}{l}\text { Not statistically } \\
\text { significant }(p=0.96)\end{array}$ \\
\hline & & & & $\begin{array}{l}\text { 2. Partial breastfeedin (i.e. } \\
\text { breastmilk and formula) }\end{array}$ & & & & \\
\hline & & & & $\begin{array}{l}\text { 3. Bottle-feeding (i.e. no } \\
\text { breastmilk at all) }\end{array}$ & & & & \\
\hline
\end{tabular}


Table 1 Japanese studies dealing with factors that influence the duration of breastfeeding (Continued)

\begin{tabular}{|c|c|c|c|c|c|c|c|c|}
\hline \multirow[t]{3}{*}{ Educational status } & \multirow[t]{3}{*}{$\begin{array}{l}\text { Otsuka et al. } \\
\text { (2008) [22] }\end{array}$} & \multirow[t]{3}{*}{ Cross-sectional } & \multirow[t]{3}{*}{262} & $\begin{array}{l}\text { 1. Exclusive Breastfeeding (i.e. } \\
\text { breastmilk only) }\end{array}$ & 40 at $4 w k s$ & \multirow[t]{3}{*}{$\begin{array}{l}1.3(0.66,2.49)(\mathrm{High} \\
\text { school or less) }\end{array}$} & \multirow[t]{3}{*}{ N/A } & \multirow[t]{3}{*}{$\begin{array}{l}\text { Not statistically } \\
\text { significant }(p=0.47)\end{array}$} \\
\hline & & & & $\begin{array}{l}\text { 2. Partial breastfeeding (i.e. } \\
\text { breastmilk and formula) }\end{array}$ & & & & \\
\hline & & & & $\begin{array}{l}\text { 3. Bottle-feeding (i.e. no } \\
\text { breastmilk at all) }\end{array}$ & & & & \\
\hline \multirow{6}{*}{$\begin{array}{l}\text { Maternal employment } \\
\text { status }\end{array}$} & \multirow{3}{*}{$\begin{array}{l}\text { Kaneko et al. } \\
\text { (2006) [20] }\end{array}$} & \multirow[t]{3}{*}{ Cross-sectional } & \multirow[t]{3}{*}{45,569} & 1. Breastfed & 25.0 at $6 \mathrm{~m}$ & \multirow{3}{*}{$\begin{array}{l}1.17(1.09,1.26) \\
\text { (Full time and child } \\
\text { care leave for } 6 \mathrm{~m} \text { or } \\
\text { more) }\end{array}$} & \multirow{3}{*}{$\begin{array}{l}1.14(1.05,1.23) \\
\text { (Full time and child } \\
\text { care leave for } 6 \mathrm{~m} \text { or } \\
\text { more) }\end{array}$} & \multirow{3}{*}{$\begin{array}{l}\text { Non-workers = } 1 \\
\text { (reference) }\end{array}$} \\
\hline & & & & 2. Only colostrum & & & & \\
\hline & & & & 3. Not breastfeed & & & & \\
\hline & \multirow{3}{*}{$\begin{array}{l}\text { Sasaki et al. } \\
\text { (2009) [23] }\end{array}$} & \multirow[t]{3}{*}{ Longitudinal } & \multirow[t]{3}{*}{908} & 1. Only breastfeeding & \multirow[t]{3}{*}{27.6 at $3-4 m$} & \multirow[t]{3}{*}{ N/A } & \multirow[t]{3}{*}{$\mathrm{N} / \mathrm{A}$} & \multirow{3}{*}{$\begin{array}{l}\text { Not statistically } \\
\text { significant }(p=0.12)\end{array}$} \\
\hline & & & & $\begin{array}{l}\text { 2. Breastmilk and } \\
\text { infant formula }\end{array}$ & & & & \\
\hline & & & & 3. Only infant formula & & & & \\
\hline \multirow[t]{9}{*}{ Multiple births } & \multirow{3}{*}{$\begin{array}{l}\text { Kaneko et al. } \\
\text { (2006) [20] }\end{array}$} & \multirow[t]{3}{*}{ Cross-sectional } & \multirow[t]{3}{*}{46,569} & 1. Breastfed & \multirow[t]{3}{*}{1.3 at $6 \mathrm{~m}$} & \multirow[t]{3}{*}{$0.05(0.03,0.09)$} & \multirow[t]{3}{*}{$0.07(0.04,0.12)$} & \multirow{3}{*}{$\begin{array}{l}\text { Single birth }=1 \\
\text { (reference) }\end{array}$} \\
\hline & & & & 2. Only colostrum & & & & \\
\hline & & & & 3. Not breastfeed & & & & \\
\hline & \multirow{3}{*}{$\begin{array}{l}\text { Yokoyama et al. } \\
\text { (2006) [21] }\end{array}$} & \multirow{3}{*}{ Cross-sectional } & \multirow[t]{3}{*}{15,262} & 1. Breastfeeding only & N/A & N/A & $\mathrm{N} / \mathrm{A}$ & Multiple births was \\
\hline & & & & $\begin{array}{l}\text { 2. Mixed-feeding (mainly } \\
\text { breast with some bottle or } \\
\text { mainly bottle with some } \\
\text { breast) }\end{array}$ & & & & $\begin{array}{l}\text { assoclated with } \\
\text { breastfeeding cessation } \\
\text { (adjusted OR = } 0.07 \\
95 \% \mathrm{Cl}=0.04,0.12 \text { ) }\end{array}$ \\
\hline & & & & $\begin{array}{l}\text { 3. Bottle feeding with } \\
\text { formula milk only }\end{array}$ & & & & \\
\hline & Ooki (2008) [24] & Cross-sectional & 4,023 & 1 Full breastfeeding & 6.6 at $6 \mathrm{~m}$ (Full & N/A & N/A & Sample were mother \\
\hline & & & & $\begin{array}{l}\text { 2. Partial breastfeeding } \\
\text { (mixed feeding) }\end{array}$ & & & & $\begin{array}{l}\text { Breastfeeding and } \\
\text { formula feeding at }\end{array}$ \\
\hline & & & & 3. Formula feeding & & & & $\begin{array}{l}6 \mathrm{~m} \text { were } 36.1 \text { and } \\
54.1 \% \text {, respectively }\end{array}$ \\
\hline Maternal smoking & Kaneko et al. & Cross-sectional & 46,324 & 1. Breastfed & 11.5 at $6 \mathrm{~m}$ & $0.40(0.31,0.95)$ & $0.44(0.34,0.57)$ & Nil smoking of the \\
\hline & (2000) [2U] & & & 2. Only colostrum & & & & $\begin{array}{l}\text { mothers and } \\
\text { fathers = } 1\end{array}$ \\
\hline & & & & 3. Not breastfeed & & & & (reference) \\
\hline & Sasaki et al. & Longitudinal & 908 & 1. Only breastfeeding & 27.6 at $3-4 m$ & N/A & $\mathrm{N} / \mathrm{A}$ & Not statistically \\
\hline & & & & $\begin{array}{l}\text { 2. Breastmilk and } \\
\text { infant formula }\end{array}$ & & & & significant $(p=0.25)$ \\
\hline & & & & 3. Only infant formula & & & & \\
\hline Delivery methods & $\begin{array}{l}\text { Nakao et al. } \\
\text { (2008) [25] }\end{array}$ & Cross-sectional & 318 & $\begin{array}{l}\text { 1. Fully breastfeeding } \\
\text { (breastmilk was given } \\
\text { and infant formula was }\end{array}$ & N/A & N/A & $1.02(0.41,2.59)$ & $\begin{array}{l}\text { Not statistically } \\
\text { significant }(p=0.96)\end{array}$ \\
\hline
\end{tabular}


Table 1 Japanese studies dealing with factors that influence the duration of breastfeeding (Continued)

Sasaki et al.
(2009) [23]

Longitudinal

Kaneko et al.

(2006) [20]

Cross-sectional

Otsuka et al.

(2008) [22]

Sasaki et al.

(2009) [23]

Current intake of

alcohols

Sasaki et al.

(2009) [23]

Longitudinal

Longitudinal

908

908

908

1. Only breastfeeding

2. Breastmilk and

infant formula

3. Only infant formula

1. Only breastfeeding

2. Breastmilk and

infant formula

3. Only infant formula

Early initiation of breastfeeding
24.5 at $6 \mathrm{~m}$

(Second times)

26.1at $6 \mathrm{~m}$ (Third

times or more)

$1.57(1.49,1.65)$
(Second times)
$1.71(1.60-1.81)$
(Third times or

$1.72(1.63,1.81)$

(Second times)

2.06 (1.91-2.22)

(Third times or

more)

N/A

N/A

was not given, regardless
N/A

N/A

N/A

N/A
Not statistically

significant $(p=0.90)$

Not statistically

significant $(p=0.14$

Primipara $=1$

(reference)

Not statistically

significant $(p=0.55)$

Not intake of

alcohols $=1$

(reference)

$(p=0.04)$

Initiating breastfeeding

within 120 minutes after

birth was positively

associated with 
Table 1 Japanese studies dealing with factors that influence the duration of breastfeeding (Continued)

\begin{tabular}{|c|c|c|c|c|c|c|c|c|}
\hline & & & & $\begin{array}{l}\text { of whether other liquids } \\
\text { and/or solid food } \\
\text { were given) }\end{array}$ & & & & $\begin{array}{l}\text { duration of } \\
\text { breastfeeding at } \\
4 \text { months }(p=0.01)\end{array}$ \\
\hline & & & & $\begin{array}{l}\text { 2. Any Breastfeeding } \\
\text { (breastmilk and infant } \\
\text { formula were given } \\
\text { regardless of whether } \\
\text { other liquids and/or } \\
\text { solid food were given) }\end{array}$ & & & & \\
\hline & & & & $\begin{array}{l}\text { 3. Infant formula } \\
\text { feeding (formula was given } \\
\text { and breast milk was not } \\
\text { given regardless } \\
\text { of whether other liquids } \\
\text { and/or solid food } \\
\text { were given) }\end{array}$ & & & & \\
\hline \multirow{6}{*}{$\begin{array}{l}\text { Intention to } \\
\text { breastfeed }\end{array}$} & \multirow{3}{*}{$\begin{array}{l}\text { Nakamura et al. } \\
\text { (2002) [26] }\end{array}$} & \multirow[t]{3}{*}{ Cross-sectional } & \multirow[t]{3}{*}{105} & 1. Breastfeeding & \multirow[t]{3}{*}{73.9 at $3 \mathrm{~m}$} & \multirow[t]{3}{*}{ N/A } & \multirow[t]{3}{*}{ N/A } & \\
\hline & & & & 2. Breast \& Bottle feeding & & & & \\
\hline & & & & 3. Artificial milk & & & & \\
\hline & \multirow[t]{3}{*}{$\begin{array}{l}\text { Otsuka et al. } \\
\text { (2008) [22] }\end{array}$} & \multirow[t]{3}{*}{ Cross-sectional } & \multirow[t]{3}{*}{262} & $\begin{array}{l}\text { 1. Exclusive Breastfeeding (i.e. } \\
\text { breastmilk only) }\end{array}$ & \multirow[t]{3}{*}{40 at $4 w k s$} & \multirow[t]{3}{*}{ N/A } & \multirow[t]{3}{*}{ N/A } & \multirow{5}{*}{$\begin{array}{l}\text { Intention to Exclusive } \\
\text { Breastfeeding = } 1 \\
\text { (reference) }\end{array}$} \\
\hline & & & & $\begin{array}{l}\text { 2. Partial breastfeeding (i.e. } \\
\text { breastmilk and formula) }\end{array}$ & & & & \\
\hline & & & & $\begin{array}{l}\text { 3. Bottle-feeding (i.e. no } \\
\text { breastmilk at all) }\end{array}$ & & & & \\
\hline \multirow[t]{2}{*}{ Maternal attachment } & \multirow{2}{*}{$\begin{array}{l}\text { Sasano et al. } \\
\text { (2005) [27] }\end{array}$} & \multirow[t]{2}{*}{ Cross-sectional } & \multirow[t]{2}{*}{182} & 1. Breastfeeding & \multirow[t]{2}{*}{53.4 at $3 \mathrm{~m}$} & \multirow[t]{2}{*}{ N/A } & \multirow[t]{2}{*}{ N/A } & \\
\hline & & & & $\begin{array}{l}\text { 2. Mix and infant formula } \\
\text { feeding }\end{array}$ & & & & \\
\hline \multirow[t]{3}{*}{ Breastmilk insufficiency } & \multirow{3}{*}{$\begin{array}{l}\text { Yoshitome et al. } \\
\text { (2003) [28] }\end{array}$} & \multirow[t]{3}{*}{ Cross-sectional } & \multirow[t]{3}{*}{246} & 1 Breastfeeding only & \multirow[t]{3}{*}{38.5 at $3-4 m$} & \multirow[t]{3}{*}{ N/A } & \multirow[t]{3}{*}{ N/A } & \\
\hline & & & & 2. Mix feeding & & & & \\
\hline & & & & 3. Infant formula only & & & & \\
\hline \multirow[t]{3}{*}{ Low birth weight } & \multirow{3}{*}{$\begin{array}{l}\text { Kaneko et al. } \\
\text { (2006) [20] }\end{array}$} & \multirow[t]{3}{*}{ Cross-sectional } & \multirow[t]{3}{*}{46,557} & 1. Breastfed & \multirow[t]{3}{*}{11.4 at $6 \mathrm{~m}$} & \multirow[t]{3}{*}{$0.46(0.42,0.51)$} & \multirow[t]{3}{*}{$0.67(0.60,0.76)$} & $\geq 2500 \mathrm{~g}=1$ (reference) \\
\hline & & & & 2. Only colostrum & & & & \\
\hline & & & & 3. Not breastfeed & & & & \\
\hline Maternal confidence & $\begin{array}{l}\text { Awano et al. } \\
\text { (2010) [29] }\end{array}$ & quasi-experimental & 117 & $\begin{array}{l}1 \text { Fully breastfeeding (no } \\
\text { formula was given) }\end{array}$ & $\begin{array}{l}72.2 \text { at } 4 \text { wks(Full } \\
\text { breastfeeding) }\end{array}$ & N/A & N/A & $\begin{array}{l}\text { Breastfeeding self-care } \\
\text { program is effect to } \\
\text { improve maternal } \\
\text { confidence }\end{array}$ \\
\hline & $\begin{array}{l}\text { Otsuka et al. } \\
\text { (2008) [22] }\end{array}$ & Cross-sectional & 262 & $\begin{array}{l}\text { 1. Exclusive Breastfeeding (i.e. } \\
\text { breastmilk only) }\end{array}$ & 40 at 4 wks & N/A & N/A & $\begin{array}{l}\text { Maternal confidence } \\
\text { level is related to their }\end{array}$ \\
\hline & & & & $\begin{array}{l}\text { 2. Partial breastfeeding (i.e. } \\
\text { breastmilk and formula) }\end{array}$ & & & & $\begin{array}{l}\text { breast milk flow. } \\
\text { berent }\end{array}$ \\
\hline
\end{tabular}


Table 1 Japanese studies dealing with factors that influence the duration of breastfeeding (Continued)

\begin{tabular}{|c|c|c|c|c|c|c|c|c|}
\hline \multirow[t]{3}{*}{ Sucking difficulty } & \multirow{3}{*}{$\begin{array}{l}\text { Yokoyama et al. } \\
\text { (2006) [21] }\end{array}$} & \multirow[t]{3}{*}{ Cross-sectional } & \multirow[t]{3}{*}{15,262} & 1. Breastfeeding only & \multirow[t]{3}{*}{ N/A } & \multirow[t]{3}{*}{ N/A } & \multirow[t]{3}{*}{ N/A } & \multirow{3}{*}{$\begin{array}{l}\text { Infants with poor sucking } \\
\text { ability are } 1.56 \text { times } \\
\text { more like to be given } \\
\text { infant formula ( } 95 \% \\
\mathrm{Cl}=1.12,2.18 \text { ) }\end{array}$} \\
\hline & & & & $\begin{array}{l}\text { 2. Mixed-feeding (mainly } \\
\text { breast with some bottle } \\
\text { or mainly bottle with } \\
\text { some breast) }\end{array}$ & & & & \\
\hline & & & & $\begin{array}{l}\text { 3. Bottle feeding with } \\
\text { formula milk only }\end{array}$ & & & & \\
\hline \multirow{6}{*}{$\begin{array}{l}\text { Support from health } \\
\text { professionals }\end{array}$} & \multirow{3}{*}{$\begin{array}{l}\text { Sasaki et al. } \\
\text { (2009) [23] }\end{array}$} & \multirow[t]{3}{*}{ Longitudinal } & \multirow[t]{3}{*}{908} & 1. Only breastfeeding & \multirow[t]{3}{*}{ N/A } & \multirow[t]{3}{*}{ N/A } & \multirow{3}{*}{$\begin{array}{l}0.83 \text { (0.61,1.12) } \\
\text { (Midwives) }\end{array}$} & \multirow{3}{*}{$\begin{array}{l}\text { Without support from } \\
\text { midwives }=1 \\
\text { (reference) }(p=0.83)\end{array}$} \\
\hline & & & & $\begin{array}{l}\text { 2. Breastmilk and } \\
\text { infant formula }\end{array}$ & & & & \\
\hline & & & & 3. Only infant formula & & & & \\
\hline & \multirow{3}{*}{$\begin{array}{l}\text { Kaneko et al. } \\
\text { (2006) [20] }\end{array}$} & \multirow[t]{3}{*}{ Cross-sectional } & \multirow[t]{3}{*}{45,569} & 1. Breastfed & \multirow[t]{3}{*}{29.7 at $6 \mathrm{~m}$} & \multirow[t]{3}{*}{$1.63(1.50,1.78)$} & \multirow[t]{3}{*}{$1.76(1.60,1.94)$} & \multirow{3}{*}{$\begin{array}{l}\text { Advice on child care } \\
\text { from birth attendant/ } \\
\text { nurse }\end{array}$} \\
\hline & & & & 2. Only colostrum & & & & \\
\hline & & & & 3. Not breastfeed & & & & \\
\hline \multirow{5}{*}{$\begin{array}{l}\text { Support from } \\
\text { husbands/partners }\end{array}$} & \multirow{3}{*}{$\begin{array}{l}\text { Kaneko et al. } \\
\text { (2006) [20] }\end{array}$} & \multirow[t]{3}{*}{ Cross-sectional } & \multirow[t]{3}{*}{45,569} & 1. Breastfed & \multirow[t]{3}{*}{21.8 at $6 \mathrm{~m}$} & \multirow[t]{3}{*}{$1.28(1.21,1.37)$} & \multirow[t]{3}{*}{$1.07(1.00,1.14)$} & \multirow{3}{*}{$\begin{array}{l}\text { Support = advice on } \\
\text { child care from } \\
\text { husbands. No } \\
\text { advice }=1 \text { (reference) }\end{array}$} \\
\hline & & & & 2. Only colostrum & & & & \\
\hline & & & & 3. Not breastfeed & & & & \\
\hline & $\begin{array}{l}\text { Ninomiya et al. } \\
\text { (1997) [30] }\end{array}$ & Cross-sectional & 264 & $\begin{array}{l}\text { 1. Breastfeeding but } \\
\text { exclusion of mix } \\
\text { breastfeeding }\end{array}$ & 37.5 at $3 \mathrm{~m}$ & N/A & N/A & $\begin{array}{l}\text { Father's attendance } \\
\text { to an antenatal class } \\
\text { is correlated to full } \\
\text { breastfeeding } \\
\text { duration ( } \mathrm{p}<0.01 \text { ). }\end{array}$ \\
\hline & $\begin{array}{l}\text { Ninomiya et al. } \\
\text { (1995) [31] }\end{array}$ & Cross-sectional & 719 & $\begin{array}{l}\text { 1. Breastfeeding but } \\
\text { exclusion of mix } \\
\text { breastfeeding }\end{array}$ & N/A & N/A & N/A & $\begin{array}{l}\text { Father's involvements } \\
\text { in childcare are } \\
\text { associated with full } \\
\text { breastfeeding } \\
\text { duration for three } \\
\text { months postpartum } \\
(p<0.05) \text {. }\end{array}$ \\
\hline \multirow{3}{*}{$\begin{array}{l}\text { Family smoking } \\
\text { environment }\end{array}$} & \multirow{3}{*}{$\begin{array}{l}\text { Kaneko et al. } \\
\text { (2006) [20] }\end{array}$} & \multirow[t]{3}{*}{ Cross-sectional } & \multirow[t]{3}{*}{45,569} & 1. Breastfed & 22.6 & $0.90(0.86,0.95)$ & $0.92(0.88,0.97)$ & Non-smoking fathers \\
\hline & & & & 2. Only colostrum & & & & (reference) \\
\hline & & & & 3. Not breastfeed & & & & \\
\hline Breastfeeding & Sasaki et al. & Longitudinal & 908 & 1. Only breastfeeding & N/A & N/A & $2.62(1.85,3.73)$ & Not breastfeeding \\
\hline auring nignt & $(2009)[23]$ & & & $\begin{array}{l}\text { 2. Breastmilk and } \\
\text { infant formula }\end{array}$ & & & & (reference) $(p<0.01)$ \\
\hline & & & & 3. Only infant formula & & & & \\
\hline
\end{tabular}


sections has increased rapidly in the past decade [40,41]. While Nakao et al. [25] and Sasaki et al. [23] analysed delivery methods as a factor that influences breastfeeding duration, the results were not significant. Associations between parity and breastfeeding duration are not consistent. In the study by Kaneko et al., multiparous women were more likely to continue Exclusive Breastfeeding for six months postpartum compared to those who were primiparous $(\mathrm{aOR}=1.72,95 \% \mathrm{CI}=1.63-1.81$ for second delivery; $\mathrm{aOR}=2.06,95 \% \mathrm{CI}=1.91-2.22$ for third delivery) [20]. However, other studies have not confirmed this association [22,23]. Parity was found to be associated with breastfeeding duration only in the cross-sectional study, with a larger sample size, but not in the cohort studies with smaller sample sizes.

A number of studies identified that mothers who intend to breastfeed' their infants at an early stage of pregnancy tended to have a longer duration of breastfeeding. A study in Okinawa found that mothers who intended to breastfeed their infants during pregnancy were more likely to continue breastfeeding at three months, compared with mothers undecided about feeding methods $\left(\mathrm{X}^{2}=28.3837, \mathrm{p}<0.01\right)$ [26]. In a further study from Tokyo and Kyoto $(n=262)$, mothers who intended to 'breastfeed', compared to those who intended to 'exclusively breastfeed' were more likely to have introduced infant formula at four weeks postpartum (OR $=8.6,95 \%$ 2.5-29.5) [22]. However a recent survey of 2,722 postpartum mothers showed that only $42 \%$ were fully breastfeeding at four weeks postpartum, although during pregnancy more than $95 \%$ had intended to breastfeed their children [42]. Mothers with positive attitudes, sentiment and confidence were also more likely to continue breastfeeding [43].

Maternal confidence in their ability to breastfeed their infants, as measured by Breastfeeding Self-Efficacy Scale-Short Form, were associated with infant feeding outcome at four weeks. Otsuka et al. showed that mothers with a lower score $(=<44)$ of breastfeeding selfefficacy were more likely to use infant formula within four weeks $(\mathrm{aOR}=3.5,95 \% \mathrm{CI}=1.8-6.6)$ [22]. In a study using a quasi-experimental method of 117 primiparous mothers, maternal confidence levels towards breastfeeding were improved by breastfeeding education, particularly focusing on self-care programs about their breasts during breastfeeding [29]. Mothers who received this education program before hospital discharge had a higher proportion of full breastfeeding at four weeks than the control group of mothers $(90 \%$ and $65 \%$, respectively, $\mathrm{p}=0.02$ ) [29]. Maternal confidence levels are also found to be associated with lower levels of perceptions of breastmilk insufficiency. Otsuka et al. reported that 'maternal perception of insufficient breastmilk' at four weeks postpartum was significantly associated with 'breastfeeding self-efficacy' level at immediate postpartum periods after birth $(\mathrm{r}=0.45, \mathrm{p}<0.001)$ [22].

Breastmilk insufficiency is a common reason given by mothers in most cultures for terminating breastfeeding and/or introducing supplementary feeding their infants [44]. A survey of 241 Japanese mothers found that at one week more than $41 \%$, and at 4 weeks $70 \%$, perceived that they had breastmilk insufficiency and had introduced infant formula [28]. While the participants in both studies voluntarily completed self-reported questionnaires, sample sizes in these studies were again small.

A cross-sectional study of 182 Japanese mothers investigated associations between levels of maternal attachment and infant feeding methods at three months of age. Sasano and Sumitani showed that mothers who were breastfeeding exclusively had a higher score for 'pleasurable interaction', a component of the maternal attachment scale, compared to those who were wholly or partly using infant formula feeding [27]. This study concluded that it is important for mothers to gain support and assistance during breastfeeding in order to increase and improve their maternal-infant bonding [27]. However this study is limited by the lack of multivariate analysis and the small sample size.

\section{Infants' attributes}

Factors relating to the infant are also important in breastfeeding, including low birth weight and multiple births. Low birth weight infants (less than $2500 \mathrm{~g}$ ) were less likely to be breastfed at six months $(\mathrm{aOR}=0.67$, 95\% CI $=0.60-0.76$ ) [20]. Similarly, Yokoyama et al. [21] found that mothers with multiple births were 2.44 times more likely to choose bottle feeding than those who had a singleton birth. However, the full breastfeeding rate of twins has not changed over 30 years with a study of 4,023 twins born between 1968 and 2003 showing that the rate of full breastfeeding at one month postpartum was steady at $16.8 \%$ between 1975 and 1984 and $16.7 \%$ between 1995 and 2003 [24].

\section{Socio-environmental attributes}

Professional support in obstetric facilities is essential for mothers to improve breastfeeding rates. Kaneko et al. showed that mothers who received advice about childcare from professionals, including birth attendant/ nurse, increased the duration of Exclusive Breastfeeding $(\mathrm{aOR}=1.76,95 \% \mathrm{CI}=1.60-1.94)$ [20]. Similarly, support from partners/fathers of infants was found to be important. In an early study investigating a relationship between fathers' attendance to an antenatal class before delivery and breastfeeding duration $(\mathrm{n}=264)$, Ninomiya et al. showed that for women (mothers) who continued 'full breastfeeding' to three months postpartum, it was 
more likely that their husbands would also have attended the classes, compared with those who ceased breastfeeding $(63.0 \%$ and $37.0 \%$ respectively, $\mathrm{p}<0.01)$ [30]. The same authors also found in another study $(\mathrm{n}=719)$ that mothers who were satisfied with the involvement of fathers in childcare tended to continue full breastfeeding at three months $(\mathrm{p}<0.05)$ [31]. These studies showed statistical significances using univariate analysis. Kaneko et al., using multivariate analysis, showed that mothers who discussed childcare with their partners were more likely to continue Exclusive Breastfeeding for six months $(\mathrm{aOR}=1.07,95 \% \mathrm{CI}=1.00-1.14)[20]$. On the other hand, there was a negative association of mothers' mothers (grandparents of infants) towards breastfeeding duration. A study reported that 'not living with their own mothers and fathers (grandparents of infants)' was positively associated with breastfeeding status at six months after birth, compared with those who lived with their parents $(\mathrm{aOR}=1.14,95 \% \mathrm{CI}=1.07-1.21)$ [20].

WHO and UNICEF encourage mothers to have skin to skin contact for at least 60 minutes immediately after birth and then to support early breastfeeding by 'rooming-in', as documented in 'The ten steps to successful breastfeeding' [45]. A study of 319 mothers who initiated breastfeeding within 120 minutes after birth, compared to those initiating later, found higher rates of full breastfeeding at four months postpartum ( $\mathrm{aOR}=2.5,95 \%$ $\mathrm{CI}=1.21-4.95)[25]$. A government survey reported that $32.4 \%$ of 2,722 mothers initiated breastfeeding within 30 minutes after delivery and $17.3 \%$ of them commenced rooming-in immediately post birth [6].

\section{Discussion}

Unlike studies from other countries [16,17], our review found that several factors, including maternal age, family income and maternal educational levels, were not associated with breastfeeding duration in Japan. Smoking status, low birth weight of infants and maternal perceptions of insufficient breastmilk supply were associated with shorter breastfeeding duration. Also, living with grandparents of infants in Japan may be an impediment to breastfeeding duration. These negative factors are keys to understanding potential targets for health promotion programs that are relevant to breastfeeding culture in Japan. One positive association that appears to differ from other countries was the association found in several studies between returning to work (child care facility provided) and continued breastfeeding [20]. This is an interesting finding that may relate to level of education and socio-economic status, but it requires further studies in different parts of the country to confirm the finding and explore the reasons for it.

Although the project, 'Healthy and Happy Family 21 (Sukoyaka Oyako 21) was undertaken by the Japanese government, a survey of 757 community centres reported that only $10 \%$ had implemented breastfeeding programs to improve breastfeeding rates at the local level [46]. Countries that have improved breastfeeding rates have usually had a national promotion program and enlist society as a whole to support breastfeeding.

While the Japanese health system puts great emphasis on healthy development and prevention, the national breastfeeding rate at one month postpartum has remained relatively unchanged between 1980 and 2005 and appears to have slightly declined in some surveys. In Australia, a country with similar health status and level of economic development to Japan, the rate of Exclusive Breastfeeding at one month postpartum was $55.8 \%$ [47], higher than Japan at $42.4 \%$. Different definitions of breastfeeding practices were also used in Australia and Japan. Australia uses the WHO definitions, while the latest Japanese survey (The National Survey of Infants Growth in 2010) used a definition of Exclusive Breastfeeding that was having breastmilk as the main source of nutrition and included having some infant formula when infants "go out" [13]. Consequently, much breastfeeding in Japan that is reported as Exclusive Breastfeeding is actually Predominant (full) or Any Breastfeeding and not Exclusive Breastfeeding according to the WHO definition. However, even though a less stringent definition has been used, there is still room for considerable improvement in Exclusive Breastfeeding rates to six months. This would be in accord with WHO policy and would optimise the health of Japanese infants.

In addition, some practices, including prelacteal feeding and supplemental feeding, which are not recommended by WHO, are still common in Japan. A crosssectional study of 1,612 postpartum mothers in Himeji city reported that the mean age of starting infant formula was 2.8 weeks, which meant that the majority were classified as Any Breastfeeding after their discharge from hospital. More than 20\% introduced infant formula within seven days after birth and this increased to almost $80 \%$ at two weeks postpartum [48]. In a study of 41 obstetric facilities in Okinawa prefecture (approximately $47 \%$ of the total number of the obstetric facilities), Nakamura showed that only 8 facilities (19.5\%) routinely gave nothing to infants after birth except breastmilk from their own mother [26]. Nineteen facilities $(46.3 \%)$ gave infant formula or glucose to infants while in hospital and five facilities (12.1\%) gave these immediately after birth as their first feed. However, some authors have argued that giving glucose water as the first feed for infants prevents neonatal hypoglycaemia that may affect the neurological problems later in life $[49,50]$. One randomized controlled trial (RCT) found that prelacteal or early supplemental feeding was associated with shorter duration of breastfeeding, although there are no 
reported studies that investigated this relationship in Japan [51]. Widespread use of infant formula is observed in Japan, which is consistent with the low proportion of BFHI accredited hospitals and obstetric clinics. By 2011, it was only $2 \%$ (61 out of over 3000 hospitals and obstetrics clinics) compared to Australia, where approximately 22\% (74 hospitals out of 335 obstetric/maternity hospitals) were BFHI certified in 2010 [52].

In Japan, infant formula is often introduced when infants are considered to have insufficient weight gain. This often occurs when mothers perceive that they have insufficient breastmilk production [53]. These mothers may feel guilty at not being able to breastfeed and encouraging these mothers to exclusively breastfeed their infants may subject mothers to added mental pressure and stress [50]. Health professionals also sometimes suggest to these mothers to add infant formula to relieve them from these pressures and stresses [54]. Some mothers misinterpret infants' behaviours as a sign of insufficient breastmilk [55]. Health professionals sometimes suggest adding infant formula based on their assessment of the adequacy of breastmilk [56]. The high rate of use of infant formula for supplementary feeding is reflected in the unchanged rate of Exclusive Breastfeeding since 1980. The routine use of supplemental and prelacteal infant formula feeds is not consistent with best infant feeding practice [57]. However, Any Breastfeeding is still better for the infant than receiving only infant formula and even if mothers use some supplemental infant formula, they should continue breastfeeding as much as possible.

There are other traditional customs related to breastfeeding practices that may hinder mothers from continuation of breastfeeding in Japan. For instance, many hospitals and clinics routinely measure infants and undertake other procedures immediately after birth, which separates them from their mothers. Several studies have argued that these neonatal procedures are barriers that unnecessarily delay early initiation of breastfeeding and would in turn affect the duration of breastfeeding [25,58]. Many hospitals and obstetric clinics also accept donations of infant formula and equipment from infant formula companies and samples are sometimes distributed to new mothers while they are in hospital. Howard et al. reported that mothers who received goods from formula companies were more likely to cease breastfeeding within two weeks after birth than those who did not receive gifts [59]. A study of 151 midwives who attended an academic conference also showed that more than $45 \%$ considered that the practice of teaching new mothers to prepare infant formula not to be an obstacle to breastfeeding and $32 \%$ stated that providing free gifts of infant formula would not impede breastfeeding practices [60].
Over the past three decades, Any Breastfeeding rates in Japan have improved. The New Guidelines on Infant Feeding developed by the Japanese government in 2007 may be assisting this improvement. The new guidelines were distributed to all community health centres, encouraging all health professionals to promote breastfeeding to mothers. Health professionals can have either a positive or negative influence on the mothers' motivation to continue breastfeeding depending on their own knowledge and attitudes and what they are prepared to promote. A study evaluating breastfeeding textbooks for Japanese midwives found that the only $40 \%$ provided accurate and consistent content on infant feeding consistent with best practice [61]. Nurses' and midwives' knowledge of breastfeeding would be expected to be mainly acquired from these textbooks. Mizuno et al. also reported that paediatricians $(n=90)$ were more aware of the importance of breastmilk than obstetricians $(n=62)$ (66\% vs. 13\%) and knowledge about the WHO marketing code was at a similar level (51\% vs. 18\%) [62]. Health professionals need to receive adequate education on breastfeeding knowledge and its benefits. This will enable them to deal with common concerns of mothers and will also protect mothers from confusion resulting from inconsistent information and advice in breastfeeding.

Other factors that affect breastfeeding duration including encouragement from grandmother(s) (especially, the maternal mother), husbands/partners, and other family members and society are important for continuing breastfeeding [63,64]. However, there have been few studies, particularly of fathers' support, in relation to breastfeeding duration in Japan, although the support of fathers is acknowledged to be important for mothers to continue breastfeeding in other countries. A study of Japanese husbands that compared them with their Canadian counterparts showed they were significantly less supportive during the postpartum period in terms of giving time for their family [65]. Without such support, some Japanese women felt more isolated, and some did not even expect their husbands to participate in childcare or home duties [65]. In Japanese culture, women have a dominant responsibility for care of children and may explain the apparent lack of interest of Japanese husbands/partners in supporting breastfeeding. The 2008 National Survey on Domestic Care reported that preparing or feeding meals including infant formula to children under one year old was third on the list of supporting tasks undertaken by fathers [66]. Yokoyama and Ooki [67] found that women who had minimal support from their spouses/partners were significantly more likely to choose formula feeding $(\mathrm{OR}=1.85,95 \% \mathrm{CI}=$ 1.38-2.48), although this study focused on mothers who had twin and multiple births. More research is needed 
on the extent of the role of fathers in supporting breastfeeding in Japan and how this could be extended. Our findings also show that support from grandmothers of infants may contribute in negative ways in breastfeeding. In other countries, the 'mother of the mother' has a positive influence on breastfeeding initiation and its continuation [68]. In Japanese culture, mothers often return to their hometown for their delivery and ask for help from their mothers during antepartum and postpartum periods [69]. When mothers have knowledge about the importance of Exclusive Breastfeeding, their daughters tended to have a longer duration of breastfeeding [68].

There have been a limited number of the studies on breastfeeding factors in Japan, and there are some inconsistent results between studies. Some areas of breastfeeding practices related to breastfeeding duration need to be further studied. For example, breast problems, including nipple trauma and sore nipples, are often given as reasons for discontinuation of breastfeeding by mothers, but there are few reported studies in Japan. There is a need for further cohort studies to resolve these inconsistencies. Most reported Japanese breastfeeding studies are cross-sectional in design with small sample sizes and unclear definitions of breastfeeding. One of the largest studies ( $n=46,569)$ by Kaneko et al. used a cross-sectional design and imprecise definitions [20]. Large cohort studies are required using standard definitions of breastfeeding and including measures of breastfeeding intention, initiation and duration, together with demographic factors, physical factors, psychological factors and other potential confounders.

\section{Conclusion}

The available studies of breastfeeding in Japan suggest that some factors related to breastfeeding duration in studies in other countries show inconsistent relationships in Japanese studies. Cultural and physical factors may be involved in breastfeeding practices, but more studies, particularly larger cohort studies, are needed to confirm any association. While Any Breastfeeding rates have improved in the last three decades, the trends in the rate of Exclusive Breastfeeding are less certain due to the use of inconsistent definitions. There is a need to standardise the way Exclusive Breastfeeding is defined in Japan. In order to further improve breastfeeding rates, there is a need for to improve the inconsistent knowledge of health professionals in Japan about infant feeding. In addition, cohort studies with larger representative samples sizes and clear definitions of breastfeeding type are needed to monitor risk factors of breastfeeding outcomes and to provide the basis for promoting breastfeeding. These will enable Japanese mothers, fathers, and health professionals to move towards the internationally recommended target of 'Exclusive Breastfeeding for the first six months of life'.

\section{Abbreviations}

BFHI: Baby friendly hospital initiative; WHO: World Health Organization; UNICEF: United Nations Children's Fund; Cl: Confidence interval; ORS: Oral rehydration solution; aOR: Adjusted odds ratio.

\section{Competing interests}

The authors declare that they have no competing interests.

\section{Authors' contributions}

MI had primary responsibility for designing the study, searching and analysing literature and drafting the manuscript. CB was involved in revising the manuscript critically for important intellectual content. KO contributed to searching literature and revising the manuscript. MM contributed to searching and analysing literature. MJ revised the manuscript. All authors have given final approval of the version to be published. All authors read and approved the final manuscript.

\section{Acknowledgements}

The authors thank Ms H Hongo for her comments.

\section{Author details}

${ }^{1}$ School of Public Health, Curtin Health Innovation Research Institute, Curtin University, GPO Box U1987, Perth, Western Australia 6845, Australia. ${ }^{2}$ Department of Community and Global Health, Graduate School of Medicine, University of Tokyo, 7-3-1, Hongo, Bunkyo-ku Tokyo 113-0033, Japan. ${ }^{3}$ School of Nursing, St. Mary College, 422, Tsubukuhonmachi, Kurume City, Fukuoka, Japan.

Received: 24 January 2012 Accepted: 16 October 2012

Published: 25 October 2012

\section{References}

1. Ip S, Chung M, Raman G, Chew P, Magula N, DeVine D, Trikalinos T, Lau J: Breastfeeding and maternal and infant health outcomes in developed countries. Evid Rep Technol Assess 2007, 153:1-186.

2. Horta BL, Bahl R, Martines JC, Victora CG: Evidence on the long-term effects of breastfeeding: systematic reviews and meta-analysis. Geneva: World Health Organization; 2007.

3. Eidelman Al, Schanler RJ, Johnston M, Landers S, Noble L, Szucs K, Viehmann L, Feldman-Winter L, Lawrence R, Kim S, Onyema N, Breastfeeding S: Breastfeeding and the use of human milk. Pediatrics 2012, 129(3):E827-E841.

4. Stuebe AM: The risks of not breastfeeding for mothers and infants. Rev Obstet Gynecol 2009, 2(4):222-231.

5. Ministry of Health Labour and Welfare: In The 2008 National Nutritional Survey: an interim report [Article in Japanese]. Edited by Ministry of Health Labour and Welfare. Tokyo: Ministry of Health Labour and Welfare; 2010:1-100.

6. Ministry of Health Labour and Welfare: In The Guidelines for support of breastfeeding and weaning [Article in Japanese]. Edited by Ministry of Health Labour and Welfare Health statistics. Tokyo: Ministry of Health Labour and Welfare; 2007:1-79

7. Segawa M: Buddhism and breastfeeding. Breastfeed Med 2008 3(2):124-128.

8. Takeuchi M: Breastfeeding in Japan: historical perspectives and current attitudes and practices. Jpn Hosp 1992, 11:79-92.

9. Palmer G: Bonyu no Seijikeizaigaku [Article in Japanese]. Tokyo: Gijyutu to Ningen; 1991.

10. Matsubara M, Yamanishi M: In Bonyuikuji no kanngogaku [Article in Japanese]. Edited by Matsubara M, Yamanishi M. Osaka: Medica Syuppan; 2007.

11. Health Labour and Welfare Statistics Association: Kokumineisenodoukou [Article in Japanese]. Tokyo: Health Labour and Welfare Statistics Association; 1998:118-119.

12. Ministry of Health, Labour and Welfare: In The 2008 National Nutritional Survey [Article in Japanese]. Edited by Ministry of Health, Labour and Welfare. Tokyo: Ministry of Health, Labour and Welfare; 2009:17-26. 
13. Minister of Health, Labour and Welfare: In The 2010 National Survey of Child Growth [Article in Japanese]. Edited by Equal Employment Children and Families Bureau. Tokyo: Minister of Health, Labour and Welfare; 2011.

14. Koyama S: Konnitino ikujibunnkani kannsuru ichikousatu [Article in Japanese]. Hokurikugakuin Junior College Bulletin 2005, 37:61-69.

15. Xu F, Binns $C$, Zheng S, Wang $Y$, Zhao $Y$, Lee A: Determinants of Exclusive Breastfeeding duration in Xinjiang, PR China. Asia Pac J Clin Nutr 2007, 16(2):316-321.

16. Scott JA, Binns CW, Oddy WH, Graham Kl: Predictors of breastfeeding duration: evidence from a cohort study. Pediatrics 2006, 117(4):e646-e655.

17. Dennis CL: Breastfeeding initiation and duration: a 1990-2000 literature review. J Obstet Gynecol Neonatal Nurs 2002, 31(1):12-32.

18. Word Health Organization: Indicators for assessing infant and young child feeding practices: part 1 Definitions: conclusions of a consensus meeting held 6-8 November 2007 in Washington D.C., USA. Geneva, Switzerland. 2007.

19. Moher D, Liberati A, Tetzlaff J, Altman DG: Preferred reporting items for systematic reviews and meta-analyses: the PRISMA Statement. Open Med 2009, 3(3):e123-e130.

20. Kaneko A, Kaneita Y, Yokoyama E, Miyake T, Harano S, Suzuki K, Ibuka E, Tsutsui T, Yamamoto Y, Ohida T: Factors associated with exclusive breast-feeding in Japan: for activities to support child-rearing with breast-feeding. J Epidemiol 2006, 16(2):57-63.

21. Yokoyama Y, Wada S, Sugimoto M, Katayama M, Saito M, Sono J: Breastfeeding rates among singletons, twins and triplets in Japan: a population-based study. Twin Res Hum Genet 2006, 9(2):298-302.

22. Otsuka K, Dennis $\mathrm{CL}$, Tatsuoka H, Jimba M: The relationship between breastfeeding self-efficacy and perceived insufficient milk among Japanese mothers. J Obstet Gynecol Neonatal Nurs 2008, 37(5):546-555.

23. Sasaki Y, Takehara K, Matsumoto A, Yoshiasa K, Shimane T, Noguchi M, Misago C: Factors for practice at the fourth month: analysis as a cross-sectional data on the longitudianl progress of mothers and child through pregnancy and delivery [Article in Japanese]. Bosei Eisei (Japan Society of Maternal Health) 2009, 50(2):396-405.

24. Ooki S: Breast-feeding rates and related maternal and infants' obstetric factors in Japanese twins. Environ Health Prev Med 2008, 13(4):187-197.

25. Nakao Y, Moji K, Honda S, Oishi K: Initiation of breastfeeding within 120 minutes after birth is associated with breastfeeding at four months among Japanese women: a self-administered questionnaire survey. Int Breastfeed J 2008, 3:1.

26. Nakamura M: Factors promoting breast feeding and nursing support [Article in Japanese]. Ryukyu Medical Journal 2002, 21(2):9-17.

27. Sasano K, Sumitani Y: An assessment of factors related to maternal attachment and nursing style of mothers with three month-old infants [Article in Japanese]. Toyama Medical and Pharmaceutical University Nursing Journal 2005, 6(1):111-121.

28. Yoshitome A, Gotoh $Y$, Tomiyasu T: Factors that negatively affected infant feeding methods within 3-4 months after birth [Article in Japanese]. Perinatal Medicine 2003, 33(8):1040-1042.

29. Awano M, Shimada K: Development and evaluation of a self care program on breastfeeding in Japan: a quasi-experimental study. Int Breastfeed J 2010, 5:9.

30. Ninomiya $T$, Deguchi $H$, Ogata M: The effect that childcare learning by fathers has on the mothers' continuance of breast feeding [Article in Japanese]. The Journal of Child Health 1997, 56(1):49-53.

31. Ninomiya T, Deguchi H, Ogata M: Maternal breast feeding and paternal roles in the family [Article in Japanese]. The Journal of Child Health 1995, 54(1):66-70.

32. Haughton J, Gregorio D, Pérez-Escamilla R: Factors associated with breastfeeding duration among Connecticut special supplemental nutrition program for Women, Infants, and Children (WIC) participants. $J$ Hum Lact 2010, 26(3):266-273.

33. Scott JA, Aitkin I, Binns CW, Aroni RA: Factors associated with the duration of breastfeeding amongst women in Perth, Australia. Acta Paediatr 1999 88(4):416-421

34. Haku M: Breastfeeding: factors associated with the continuation of breastfeeding, the current situation in Japan, and recommendations for further research. J Med Invest 2007, 54(3-4):224-234

35. Arthur CR, Saenz RB, Replogle WH: The employment-related breastfeeding decisions of physician mothers. J Miss State Med Assoc 2003, 44(12):383-387.
36. Chatman LM, Salihu HM, Roofe ME, Wheatle P, Henry D, Jolly PE: Influence of knowledge and attitudes on practice among rural Jamaican mothers. Birth 2004, 31(4):265-271

37. Giglia RC, Binns CW, Alfonso H: Maternal cigarette smoking and breastfeeding duration. Acta Paediatr 2006, 95(11):1370-1374.

38. Haku M, Ohashi K: Pursuit factors limiting breastfeeding continuation using Orem's dependent care model [Article in Japanese]. Journal of Japan Academy of Midwifery 2004, 18:6-18

39. Kaneita Y, Yokoyama E, Miyake T, Harano S, Asai T, Tsutsui T, Ibuka E, Suzuki K, Kaneko A, Sone T, Takemura S, Kawahara K, Ohida T: Epidemiological study on passive smoking among Japanese infants and smoking behavior of their respective parents: a nationwide cross-sectional survey. Prev Med 2006, 42(3):210-217.

40. Qiu L, Binns C, Zhao Y, Lee A, Xie X: Breastfeeding following caesarean section in Zhejiang province: public health implications. Asia Pac J Public Health 2008, 20(Suppl):220-227

41. Betran AP, Merialdi M, Lauer JA, Bing-Shun W, Thomas J, Van Look P Wagner M: Rates of caesarean section: analysis of global, regional and national estimates. Paediatr Perinat Epidemiol 2007, 21(2):98-113.

42. Equal Employment Children and Families Bureau: The 2005 Infant and Young Child Nutrition Survey [Article in Japanese]. Tokyo: Japanese Ministry of Health, Labour and Welfare; 2006.

43. Bakoula C, Veltsista A, Prezerakou A, Moustaki M, Fretzayas A, Nicolaidou P: Working mothers breastfeed babies more than housewives. Acta Paediatr 2007, 96(4):510-515.

44. Gatti L: Maternal perceptions of insufficient milk supply in breastfeeding. J Nurs Scholarsh 2008, 40(4):355-363.

45. UNICEF/WHO: Baby Friendly Hospital Initiative, revised, updated and expanded for integrated care, Section 1, Background and Implementation, Preliminary Version. UNICEF/WHO; 2006. http://www.unicef.org/nutrition/files/ BFHI_Revised_Section1.pdf

46. Horiuchi T, Yoda T, Hashimoto T: In Starting prevention of childrearing anxiety in perinatal period: breastfeeding support in health center [Article in Japanese]. Edited by Nakamura T. Toyko: Japanese Ministry of Health, Labour and Welfare Health Science Research 2003; 2004

47. Australian Institute of Health and Welfare: The 2010 Australian National Infant Feeding Survey: Indicator results. Canberra: AlHW; 2011. Report No.: Cat. No. PHE 156.

48. Inoue M: Breastfeeding and perceptions of breast shape changes in Australian and Japanese women, PhD thesis.: Curtin University, School of Public Health; 2012

49. Boluyt N, van Kempen A, Offringa M: Neurodevelopment after neonatal hypoglycemia: a systematic review and design of an optimal future study. Pediatrics 2006, 117(6):2231-2243.

50. Nakai H, Hamasaki M: The risk-free interpretation of "Ten Steps to Successful Breastfeeding" [Article in Japanese]. J Natl Inst Public Health 2009, 58(1):51-55.

51. Martin-Calama J, Bunuel J, Valero MT, Labay M, Lasarte JJ, Valle F, de Miguel C: The effect of feeding glucose water to breastfeeding newborns on weight, body temperature, blood glucose, and breastfeeding duration. J Hum Lact 1997, 13(3):209-213.

52. Baby Friendly Hospital Initiative Australia: http://www.babyfriendly.org.au

53. Binns CW, Scott JA: Breastfeeding: reasons for starting, reasons for stopping and problems along the way. Breastfeed Rev 2002, 10(2):13-19.

54. Hattori R, Horiuchi H, Nunohara K, Taniguchi M: Report of breastfeeding support in Gifu prefecture [Article in Japanese]. Gifu College of Nursing Bulletin 2006, 6(2):59-63.

55. Yamamoto $H$, Tanak $M$, Takano M: The perceptions of breastfeeding in mothers who believe that their breast milk is insufficient for their baby [Article in Japanese]. Maternal Health 2009, 50(1):110-117.

56. Kato N: Recent research on growth and feeding method in infancy and childhood [Article in Japanese]. Journal of National Institute of Public Health 1998, 47(3):226-236.

57. Saadeh $\mathrm{R}$, Akre J: Ten steps to successful breastfeeding: a summary of the rationale and scientific evidence. Birth 1996, 23(3):154-160.

58. Rowe-Murray HJ, Fisher JRW: Baby friendly hospital practices: cesarean section is a persistent barrier to early initiation of breastfeeding. Birth 2002, 29(2):124-131.

59. Howard CR, Howard F, Lawrence R, Andresen E, DeBlieck E, Weitzman M: Office prenatal formula advertising and its effect on breast-feeding patterns. Obstet Gynecol 2000, 95(2):296-303. 
60. Awano M, Sekizuka M, Shimada K, Sakai A: Relations with outlook on breastfeeding of her mothers [Article in Japanese]. Journal of Japan Academy of Midwifery 2003, 16:172-173.

61. Kaso M, Miyamoto K, Koyama E, Nakayama T: Breastfeeding information in midwifery textbooks in Japan: content analysis with evaluation standards based on delphi method. J Hum Lact 2011, 27(4):367-377.

62. Mizuno K, Miura F, Itabashi K, Macnab I, Mizuno N: Differences in perception of the WHO International Code of Marketing of Breast Milk Substitutes between pediatricians and obstetricians in Japan. Int Breastfeed J 2006, 1:12.

63. Sikorski J, Renfrew MJ, Pindoria S, Wade A: Support for breastfeeding mothers: a systematic review. Paediatr Perinat Epidemiol 2003, 17(4):407-417.

64. Ingram J, Johnson D, Hamid N: South Asian grandmothers' influence on breast feeding in Bristol. Midwifery 2003, 19(4):318-327.

65. Steinberg S, Kruckman L: Reinventing fatherhood in Japan and Canada. Soc Sci Med 2000, 50(9):1257-1272.

66. National Institute of Population and Social Security Research: The Fourth National survey on domestic cares: trends [Article in Japanese]. Tokyo: National Institute of Population and Social Security Research; 2008. http://www.ipss.go.jp/site-ad/index_Japanese/ps-katei-index.htm.

67. Yokoyama Y, Ooki S: Breast-feeding and bottle-feeding of twins, triplets and higher order multiple births. Nippon Koshu Eisei Zasshi 2004, 51(11):969-974

68. Ekstrom A, Widstrom AM, Nissen E: Breastfeeding support from partners and grandmothers: perceptions of Swedish women. Birth 2003, 30(4):261-266.

69. Kobayachi Y, Chen S-J: Experience of woman's peripartum homestay with her parents and the cultivating of nurturance [Article in Japanese]. Hokkaido University Collection of Scholarly and Academic Papers 2008, 106:119-134.

doi:10.1186/1746-4358-7-15

Cite this article as: Inoue et al:: Infant feeding practices and breastfeeding duration in Japan: A review. International Breastfeeding Journal 2012 7:15.

\section{Submit your next manuscript to BioMed Central and take full advantage of:}

- Convenient online submission

- Thorough peer review

- No space constraints or color figure charges

- Immediate publication on acceptance

- Inclusion in PubMed, CAS, Scopus and Google Scholar

- Research which is freely available for redistribution 